\title{
Inclination effects in T Tauri star spectra
}

\author{
Immo Appenzeller ${ }^{1}$ and Claude Bertout ${ }^{2}$ \\ 1 Landessternwarte, Königstuhl 12, 69117 Heidelberg, Germany \\ e-mail: iappenze@lsw.uni-heidelberg.de \\ 2 Institut d'Astrophysique, 98bis Bd. Arago, 75014 Paris, France \\ e-mail: claude. bertout@obspm. fr
}

Received 28 June 2013 / Accepted 25 July 2013

\begin{abstract}
Context. Because of the presence of rotation and accretion disks, classical T Tauri stars have symmetry planes that are normally inclined relative to the plane of the sky. The inclination angles affect the observed spectral properties of these objects.

Aims. We study the influence of the inclination angles on classical T Tauri star spectra in an empirical manner.

Methods. Published inclination angles, derived from the stellar photospheric rotation or from spatially resolved circumstellar disk observations, are compared with various observed spectral properties, and correlations are established and investigated.

Results. Inclinations derived from the stellar rotation are found to be much less accurate than the published disk inclinations, and no significant correlations between spectral properties and inclinations based on rotation data could be detected. In contrast, significant correlations are found between the disk inclination angles and the apparent velocities observed for the forbidden emission lines and the wind absorption features of permitted lines. These data support the assumption of cone-like polar winds with opening angles smaller than $\approx 45^{\circ}$. Other spectral features show weaker or no inclination dependence. Using these results, the true (deprojected) flow velocities of the polar winds are derived for the investigated sample of T Tauri stars. Deprojected wind-ejection velocities appear to differ by a factor of two among the stars in our sample, which spans a range of mass-loss rates from $10^{-10} M_{\odot} / \mathrm{yr}$ to $3 \times 10^{-7} M_{\odot} / \mathrm{yr}$.
\end{abstract}

Key words. stars: formation - stars: pre-main sequence - stars: rotation - stars: winds, outflows

\section{Introduction}

Classical T Tauri stars (CTTSs) are low-mass pre-main sequence objects with active accretion disks (e.g., Bertout et al. 1988). The accretion flow to the stellar surface of CTTSs is known to take place along magnetic flux tubes that are anchored to the central stars (e.g., Camenzind 1990). The theory of the formation of low-mass stars predicts that the rotational equatorial planes of the stars and the planes of the accretion disks are similar (see, e.g., Yorke et al. 1993), although the symmetry planes of the magnetic fields may differ (e.g., Bouvier et al. 2007; O'Sullivan et al. 2005). Theoretical models of the star-disk systems and their line-emitting regions reproduce the basic observed properties of the CTTSs fairly well (see, e.g., Hartmann et al. 1994; Kurosawa et al. 2011).

However, the details of the observed spectra are expected to depend on the inclination angles of the individual stars. In addition to the inclination-dependent rotational broadening of the photospheric absorption lines, major effects on the emission line profiles and the strength and width of selected lines have been theoretically predicted (e.g., Muzerolle et al. 2001; Kurosawa et al. 2006). But comparisons of the theoretically predicted inclination dependence with observed spectra have so far led to inconclusive results (e.g., Edwards et al. 1994; Kwan et al. 2007). Therefore, in the present study we identify inclinationdependent spectral features in CTTS spectra in a purely empirical way by comparing observed spectral properties with inclinations inferred from observations. As input data we use published empirically derived inclinations, which are based either on the rotational properties of the corresponding stars or on spatially resolved observations of their circumstellar disks.

\section{The database}

This study is based on a sample of 45 CTTSs with well studied spectra, for which direct or indirect inclination information is available in the literature. A list of these stars and some relevant spectral data are given in Table 1. In 23 cases, accurate inclinations derived from spatially resolved circumstellar disks are given. For 21 stars we include inclination values derived by comparing projected rotational velocity values $v \sin i$ with the rotational periods observed for the same stars. Since we found a tight correlation between disk inclinations and the velocities of strong forbidden lines, we supplemented our sample of stars with published inclination data by 11 CTTSs with prominent forbidden line emission. In Table 1 these stars are listed without inclination values. Of course, these stars could not contribute to establishing inclination-dependent correlations, but they allowed us to increase the statistical significance of the correlations between the different inclination-sensitive spectral features discussed in Sect. 4.

Two of the stars without direct inclination information (GG Tau and UY Aur) are members of close multiple systems where the inclinations of circumbinary disks or rings are known. These inclinations are not listed in Table 1 because there are close young binaries with misaligned disks (Roccatagliata et al. 2011). Thus, inclinations of the circumbinary disks may not be representative of the circumstellar disk inclinations, which in both cases are known to be present in these systems. Also omitted in Table 1 are the published disk inclination values of the stars DN Tau, DO Tau, and DQ Tau. Although inclination values for the circumstellar disks of these objects are quoted in the Catalog of Resolved Disks, which is maintained 
Table 1. CTTSs used for this study, listed following the order of the Herbig-Bell catalog of Orion population stars (HBC).

\begin{tabular}{|c|c|c|c|c|c|c|c|c|c|c|c|c|}
\hline $\mathrm{HBC}$ & Object & $\begin{array}{l}i_{\text {rot }} \\
\text { deg }\end{array}$ & Ref. & $\begin{array}{l}i_{\text {disk }} \\
\text { deg }\end{array}$ & Ref. & $\begin{array}{c}V \mathrm{be}[\mathrm{O} \mathrm{I}] \\
\mathrm{km} \mathrm{s}^{-1}\end{array}$ & $\begin{array}{l}V \mathrm{~b}[\mathrm{~N} \mathrm{II}] \\
\mathrm{km} \mathrm{s}^{-1}\end{array}$ & Ref. & $\begin{array}{c}F W H M(\mathrm{H} \alpha) \\
\mathrm{km} \mathrm{s}^{-1}\end{array}$ & $\begin{array}{c}F W Q M(\mathrm{He} \mathrm{I}) \\
\mathrm{km} \mathrm{s}^{-1}\end{array}$ & $\begin{array}{c}\mathrm{BAc}(\mathrm{H} \alpha) \\
\mathrm{km} \mathrm{s}^{-1}\end{array}$ & $\begin{array}{c}\mathrm{BAe}(\mathrm{He} \mathrm{I}) \\
\mathrm{km} \mathrm{s}^{-1}\end{array}$ \\
\hline 25 & CW Tau & & & & & -147 & -109 & 12 & 465 & 290 & -110 & $\mathrm{w}$ \\
\hline 26 & FP Tau & & & & & -86 & & 10 & 233 & 122 & -47 & -84 \\
\hline 28 & CY Tau & 72 & 1 & 31 & 18 & -138 & & 10 & 189 & 91 & $\mathrm{w}$ & -161 \\
\hline 30 & DD Tau & & & & & -79 & -80 & 10 & 195 & 69 & -50 & -58 \\
\hline 32 & BP Tau & 32 & 2 & 36 & 18 & -31 & & 10 & 315 & 70 & $\mathrm{w}$ & $\mathrm{w}$ \\
\hline 33 & DE Tau & 57 & 2 & & & -148 & & 10 & 300 & 125 & -55 & $\mathrm{w}$ \\
\hline 34 & RY Tau & & & 66 & 19 & -115 & & 12 & 450 & w & -40 & \\
\hline 35 & T Tau N & 15 & 1 & 20 & 6 & -180 & & 15 & 275 & 225 & -190 & \\
\hline 36 & DF Tau & 50 & 2 & & & -103 & -84 & 12 & 295 & 75 & -63 & -163 \\
\hline 37 & DG Tau & 58 & 2 & 32 & 18 & -276 & -219 & 10 & 225 & 285 & -200 & -383 \\
\hline 38 & DH Tau & 58 & 1 & & & -50 & & 12 & 167 & & w & \\
\hline 43 & UX Tau A & 60 & 1 & 46 & 23 & & & & 125 & W & -155 & -95 \\
\hline 45 & DK Tau & 44 & 2 & & & -138 & & 10 & 320 & 65 & -45 & -209 \\
\hline \multirow[t]{2}{*}{48} & HK Tau B & & & 85 & 16 & -22 & -17 & 13 & 37 & 51 & $\mathrm{w}$ & \\
\hline & HH $30^{*}$ & & & 82 & 18 & -19 & -12 & 13 & 50 & 57 & $\mathrm{w}$ & \\
\hline 49 & HL Tau & & & 40 & 24 & -198 & -171 & 13 & 239 & 275 & -148 & -300 \\
\hline 50 & XZ Tau & & & & & -133 & -75 & 12 & 110 & & w & -177 \\
\hline 52 & UZ Tau E & & & 56 & 25 & -155 & -91 & 12 & 194 & 109 & -44 & -110 \\
\hline 54 & GG Tau & 56 & 2 & & & -138 & & 10 & 240 & 190 & -100 & $\mathrm{w}$ \\
\hline 56 & GI Tau & 54 & 1 & & & -69 & & 10 & 165 & 68 & -45 & $\mathrm{w}$ \\
\hline 57 & GK Tau & 46 & 2 & & & -35 & & 10 & 300 & 45 & -30 & -77 \\
\hline 58 & DL Tau & 78 & 2 & 40 & 18 & -203 & -188 & 10 & 260 & 281 & -150 & -191 \\
\hline 60 & HN Tau & & & & & -133 & -81 & 12 & 289 & 150 & $\mathrm{w}$ & $\mathrm{w}$ \\
\hline 61 & CI Tau & & & 46 & 25 & -179 & & 10 & 228 & 202 & -113 & -167 \\
\hline 63 & AA Tau & 69 & 2 & 75 & 26 & -55 & -41 & 10 & 210 & 75 & -20 & -70 \\
\hline 65 & DN Tau & 30 & 2 & & & -48 & & 10 & 195 & 45 & -58 & w \\
\hline 67 & DO Tau & & & & & -121 & & 10 & 200 & 58 & -100 & -158 \\
\hline 418 & HV Tau C & & & 84 & 17 & -41 & -25 & 13 & 98 & 109 & $\mathrm{w}$ & \\
\hline 72 & DQ Tau & & & & & -52 & & 10 & 240 & 115 & $\mathrm{w}$ & w \\
\hline 74 & DR Tau & 15 & 4 & 20 & 7 & -186 & -166 & 10 & 175 & 145 & -225 & -330 \\
\hline 75 & DS Tau & & & & & -72 & & 10 & 300 & 50 & -40 & -140 \\
\hline 76 & UY Aur & & & & & -103 & & 10 & 200 & 68 & $\mathrm{w}$ & -135 \\
\hline 77 & GM Aur & 90 & 1 & 52 & 18 & -19 & & 10 & 315 & 140 & -75 & \\
\hline 80 & RW Aur A & 37 & 2 & & & -221 & & 10 & 490 & 125 & -75 & \\
\hline 85 & GW Ori & 30 & 5 & 10 & 7 & & & & 180 & & $\mathrm{w}$ & -379 \\
\hline 250 & GQ Lup & 27 & 3 & & & -38 & & 22 & 297 & 67 & -13 & \\
\hline \multirow[t]{2}{*}{251} & RU Lup & & & 28 & 7 & -171 & -173 & 11 & 258 & 220 & $\mathrm{w}$ & \\
\hline & KH15D & & & 84 & 14 & -35 & & 14 & 121 & & -7 & \\
\hline 568 & TW Hya & 18 & 9 & 8 & 8 & -12 & & 20 & 190 & 129 & -45 & -260 \\
\hline 578 & VZ Cha & & & & & -16 & & 21 & 166 & 74 & $\mathrm{w}$ & \\
\hline 590 & HM 32 & & & & & -14 & & 11 & 173 & 58 & $\mathrm{w}$ & \\
\hline 254 & AS $205 \mathrm{~A}$ & & & 47 & 25 & -237 & & 11 & 183 & 269 & -180 & \\
\hline 286 & $\mathrm{~S}$ CrA N & & & $<45$ & 7 & -139 & & 11 & 426 & 120 & -85 & \\
\hline 291 & VV CrA & & & & & -257 & -175 & 11 & 218 & 226 & -310 & \\
\hline 292 & AS $353 \mathrm{~A}$ & & & 20 & 27 & -352 & & 10 & 275 & 335 & -250 & -279 \\
\hline
\end{tabular}

Notes. The first column gives the HBC object number. The following columns give the most common object name, the inclination derived from the stellar rotation $\left(i_{\text {rot }}\right)$ and the corresponding reference, the inclination derived from the disk aspect angle $\left(i_{\text {disk }}\right)$ and the corresponding reference, the radial velocity of the blue edge (measured at $25 \%$ intensity) of the $630 \mathrm{~nm}$ [O I] line, the velocity of the blue peak of the [N II] $658.3 \mathrm{~nm}$ line, the reference to the forbidden-line data, the H $\alpha$ FWHM line width, the FWQM width of the $587.6 \mathrm{~nm}$ He I line, the central velocity of the blueshifted wind absorption feature in the $\mathrm{H} \alpha$ emission line $(\mathrm{BAc}(\mathrm{H} \alpha)$ ), and the blue edge (measured at $25 \%$ depth) of the blueshifted wind absorption feature of the $1083 \mathrm{~nm} \mathrm{He} \mathrm{I}$ line $(\mathrm{BAe}(\mathrm{HeI}))$. w indicates that the corresponding feature could not be detected or measured.

References. (1) Bouvier et al. (1995); (2) Bouvier (2004, priv. comm.); (3) Broeg et al. (2007); (4) Alencar et al. (2001); (5) Shevchenko et al. (1998); (6) Ratzka et al. (2009); (7) Schegerer et al. (2009); (8) Akeson et al. (2011); (9) Alencar \& Batalha (2002); (10) Hartigan et al. (1995); (11) Hamann (1994); (12) Hirth et al. (1997); (13) Appenzeller et al. (2005); (14) Hamilton et al. (2003); (15) Böhm \& Solf (1994); (16) Stapelfeldt et al. (1998); (17) Stapelfeldt et al. (2003); (18) Guilloteau et al. (2011); (19) Isella et al. (2010); (20) Herczeg et al. (2007); (21) Krautter et al. (1990); (22) Appenzeller \& Wagner (1989); (23) Tanii et al. (2012); (24) Kwon et al. (2011); (25) Andrews \& Williams (2007); (26) Cox et al. (2013); (27) Curiel et al. (1997). 
by Karl Stapelfeldt ${ }^{1}$, the disks of these three stars are only marginally resolved. Their inclinations are therefore correspondingly uncertain. In one case (AS 353A), the disk inclination has been derived indirectly from the well determined inclination of the associated jet, assuming that the jet propagates in a direction that is normal to the disk plane (as observed in all CTTSs for which this information exists).

For all inclination values listed in Table 1 the sources are given in the corresponding reference columns. Generally the source giving the lowest observational error is quoted. Usually this is also the most recent derivation. In many cases, earlier derivations are mentioned in the references listed in Table 1. Additional references on earlier inclination measurements can be found in the extensive literature lists of the Catalog of Resolved Disks cited above.

The references cited in Table 1 give mean errors of the listed disk inclinations between 1 degree and 6 degrees, with an average value of 2.2 degrees. Uncertainties of the disk inclination derivations of this order are confirmed by comparing independent derivations by different authors and by measurements obtained at different wavelengths.

The accuracy of the inclinations derived from the stellar rotation is much lower. As described and discussed first by Weaver (1987) the rotational inclinations are derived by comparing projected rotational velocities $v \sin i$, derived from photospheric absorption-line profiles, with the equatorial rotational velocities $v_{\mathrm{E}}$. For $v_{\mathrm{E}}$ we have

$v_{E}=2 \pi R P^{-1}$,

where $R$ is the stellar radius, and $P$ the observed rotation period. The inclination then follows from the relation

$i=\arcsin \left[\right.$ const. $\left.\times(v \sin i) P R^{-1}\right]=\arcsin Q$,

where $Q=$ const. $\times(v \sin i) P R^{-1}$. The error $\Delta i$ of the inclination angle then becomes

$\Delta i=\frac{\partial i}{\partial Q} \Delta Q=\left(1-Q^{2}\right)^{-1 / 2} \Delta Q$

For low values of $i$, the error of $i$ is approximately proportional to the error of $Q$, but for $Q \rightarrow 1$ (i.e. $i \rightarrow \pi / 2$ ), the error becomes very large.

Inclination estimates from $v \sin i$ are also prone to systematic errors if additional line broadening effects are not properly taken into account. If additional broadening effects are overlooked, the evaluation of the line profiles tends to result in inclination angles that are systematically too large.

Of the observational input parameters of Eq. (2), the values $v \sin i$ and $P$ can be measured fairly precisely. However, the derivation of the stellar radius $R$ depends on parameters, such as the luminosity, the effective temperature, the reddening, and the veiling of the stars, which are often not very well known for CTTSs. Therefore, stellar radii of CTTSs derived by different authors typically differ by factors of two (see, e.g., Johns-Krull \& Gafford 2002). Even with $R$ being uncertain by that amount, for small $i$ a useful approximation of the inclination can still be estimated from stellar rotation data. But for inclinations $i>30$ degrees, the errors of $i$ derived from the rotation can approach $\pi / 4$. In this case the inclination essentially becomes undetermined.

In addition to observed inclinations, we present in Table 1 velocity data derived from the profiles of selected emission lines. For the forbidden lines, we provide individual references in the

\footnotetext{
1 See http://circumstellardisks.org
}

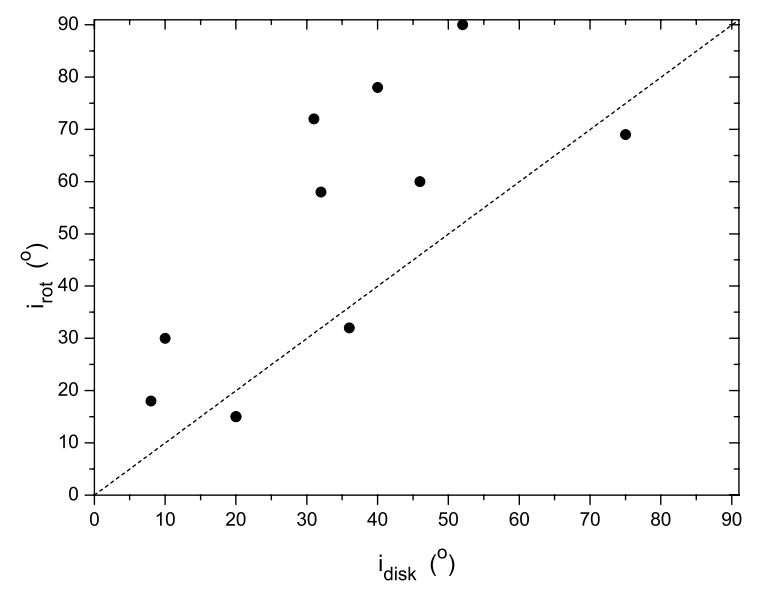

Fig. 1. Inclination angles $i_{\text {rot }}$ derived from the stellar rotation as a function of the circumstellar disk inclinations $i_{\text {disk }}$ of the corresponding objects.

column following the $[\mathrm{N} \mathrm{II}]$ data. The mean errors of the forbidden line velocity data range between $2 \mathrm{~km} \mathrm{~s}^{-1}$ and about $10 \mathrm{~km} \mathrm{~s}^{-1}$, with an average of about $6 \mathrm{~km} \mathrm{~s}^{-1}$. The information on the profiles of the $\mathrm{H} \alpha$ and $\mathrm{He}$ I $587.6 \mathrm{~nm}$ lines was in most cases taken from Alencar \& Basri (2000). For the objects not observed by these authors, the following sources were used: Beristain et al. (2001) for FP Tau, CY Tau, DD Tau, GI Tau, and HN Tau; Appenzeller et al. (2005) for HK Tau B, HH30*, and HV Tau C; Krautter et al. (1990) for VZ Cha and S CrA; Appenzeller \& Wagner (1989) for GQ Lup; Alencar \& Batalha (2002) for TW Hya; Hamann \& Persson (1992) for HM 32; Appenzeller et al. (1986) for VV CrA; Hirth et al. (1997) for $\mathrm{H} \alpha$ of DD Tau, DH Tau, and HN Tau; Boesgaard (1984) for H $\alpha$ of RU Lup; Hamilton et al. (2003) for H $\alpha$ of KH15D; Stempels \& Piskunov (2002) for He I $587.6 \mathrm{~nm}$ of RU Lup. The edge velocities of the He I $1083 \mathrm{~nm}$ absorption feature in the last column of Table 1 are based on the profiles published by Edwards et al. (2006). When spectra were not available in digital form, the corresponding features were measured in the published printed figures. The resulting accuracy (m.e. $\approx 10 \mathrm{~km} \mathrm{~s}^{-1}$ ) is adequate for the statistical purposes of this study. For the blueshifted [N II] emission peak and the wind absorption feature of the $\mathrm{H} \alpha$ line, we present central velocities. For [O I] $630 \mathrm{~nm}$ (where the blueshifted peak is often not well defined) and for the He I $1083 \mathrm{~nm}$ absorption feature, we give edge velocities, as defined in the notes to Table 1 . This has to be taken into account when the velocities are compared.

\section{Comparison of rotational and disk inclinations}

The spectral data given in Table 1 were used to search for possible correlations between the inclination and spectral properties. Initially this search was carried out separately for the inclinations based on the stellar rotation and for the inclinations of the circumstellar disks. However, for the inclination angles derived from the rotational data, no significant correlations could be detected for any of the tested spectral features. On the other hand, correlations were found, when the same spectral features were compared with the disk inclinations.

Therefore, in a next step, we used the 11 objects in Table 1 for which inclinations derived from $v \sin i$ and disk inclinations are both available to compare the inclinations derived by the two methods. As illustrated by Fig. 1, the inclinations derived by the two methods are correlated for this sample, but are clearly not identical. The mean difference between the inclinations derived 
by the two methods is about $19^{\circ}$. More critical is that the inclinations derived from $v \sin i$ are systematically larger (by about the same amount). In three cases with $i>30^{\circ}$, the difference is about $40^{\circ}$, i.e., close to $\pi / 4$. The average differences between the inclination angles derived by the two methods greatly exceed the observational errors given for the disk inclinations, but (in view of the errors discussed in Sect. 2) such deviations are expected for the rotation-based inclinations. Together with the fact that no correlations between the rotation-based inclinations and the spectral properties could be detected, we regard the poor agreement between the angles indicated in Fig. 1 as evidence that the rotation-based inclinations are, at present, not accurate and reliable enough for statistical studies. Therefore, the following sections will be based essentially on the disk-inclination values.

\section{Correlations between the disk inclinations and spectral properties}

Among the spectral features for which a correlation with inclination has been predicted most frequently are the emission lines and absorption features that are formed in the winds of the CTTSs. This applies in particular to the broad or high-velocity component of the forbidden-line profiles (see, e.g., Hartigan et al. 1995) and to the blueshifted absorption components of the hydrogen and helium permitted lines (e.g., Kurosawa et al. 2006; Beristain et al. 2001). In the following, we investigate in turn the forbidden and permitted line profiles.

\subsection{Forbidden lines}

The forbidden lines in CTTS spectra often have complex profiles, which vary greatly between individual objects and between different lines. However, in many cases the profiles can be deconvolved into a narrow or low-velocity component (LVC) with a typical FWHM of about $50 \mathrm{~km} \mathrm{~s}^{-1}$ (see Hirth et al. 1997), and a broader and usually blueshifted high-velocity component (HVC).

The HVCs are generally assumed to originate in fast outflows, which continue into the interstellar space as jets. Among the lines where the broad forbidden-line component is usually most conspicuous are the [N II] lines at $654.8 \mathrm{~nm}$ and $658.3 \mathrm{~nm}$. In many cases, the [N II] line profiles have a well defined blueshifted peak. The absolute values of the peak velocities are usually regarded as a mean for the projected wind velocities of the [N II] emitting volumes. If available, the measured velocities of the blue [N II] $658.3 \mathrm{~nm}$ peaks are listed in Table 1 .

At high inclinations, the blueshifted peak tends to merge with the low-velocity peak, and for edge-on objects the blueshifted peak can often not be separated from the narrow, low-velocity component. Among the objects in Table 1, this applies to HK Tau B and HH 30*. In these cases we include in Table 1 the highest value of the HVC-peak blueshift that appears to be compatible with the blue wing of the total [N II] profile. Since the corresponding velocity limits are close to zero (but - by definition - cannot be $>0$ ), they are, within the accuracy of this study, adequate approximations of the blueshifted peak velocities.

Resolved jet flows of CTTSs often form narrow and almost linear cones. Assuming that the unresolved inner [N II] flows have the same geometry, we may expect that the observed wind velocities are proportional to $\cos i$. Therefore, in Fig. 2 we plot the projected wind velocities, as inferred from the velocities of the blue $[\mathrm{N} \mathrm{II}]$ peaks, as a function of $\cos \left(i_{\text {disk }}\right)$. The figure

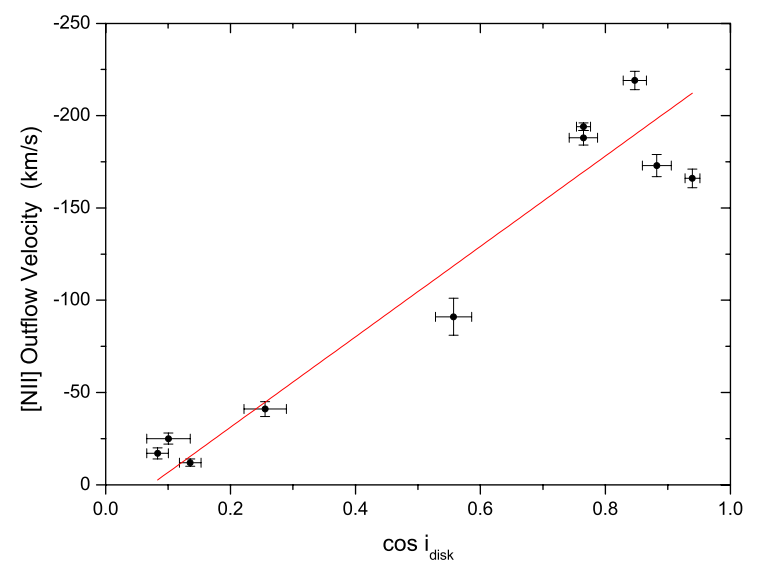

Fig. 2. Projected wind velocities inferred from the blueshifted peaks of the [N II] $658.3 \mathrm{~nm}$ lines as a function of the cosine of the disk inclination. Also included is the corresponding linear regression line.

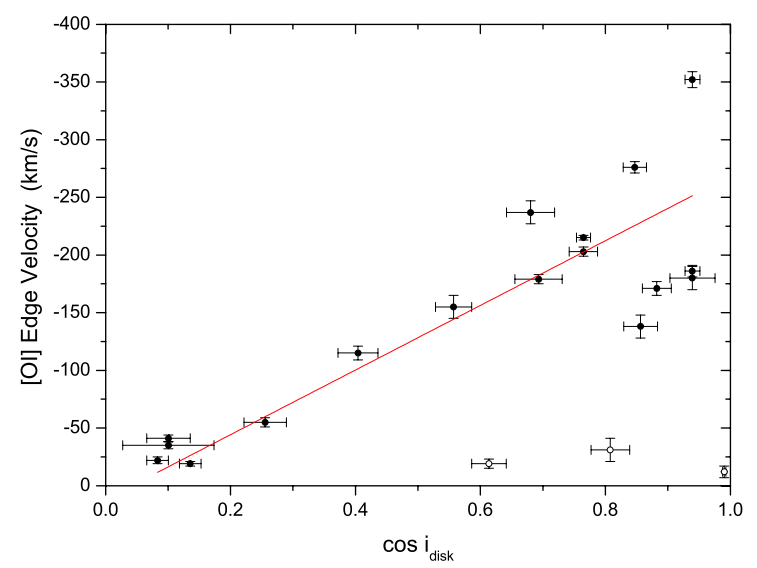

Fig. 3. Projected wind velocities inferred from the blue edges of the [O I] $630 \mathrm{~nm}$ lines as a function of the cosine of the disk inclination and the corresponding linear regression line. The open symbols refer to the three "weak-wind" objects BP Tau, GM Aur, and TW Hya.

shows that the observed [N II] wind velocities are clearly correlated with the disk inclinations. The correlation's adjusted coefficient of determination (which takes the error bars into account) is $\bar{R}=0.95$. Moreover, the plot supports the assumption that the cos $i$-law provides a good approximation. Most of the variation in the observed velocity can obviously be explained by the varying inclination.

The projection effect is also expected to influence the apparent velocity observed in the [O I] $630 \mathrm{~nm}$ line, which forms under similar conditions to the [N II] line. The [O I] line tends to be stronger and is present in the spectra of practically all stars listed in Table 1. However, compared to [N II], the oxygen line has the disadvantage of often lacking a distinct blue peak, although a broad line component is usually present in the form of an extended blue wing. As a measure of the projected (maximal) wind velocity, for this line we therefore used the blue edge of the total line profile, measured at $25 \%$ intensity. In Fig. 3 this quantity is plotted as a function of $\cos \left(i_{\text {disk }}\right)$. Again a clear correlation is observed. However, three objects, BP Tau, GM Aur, and TW Hya fall well below the relation defined by all other stars. Therefore, these three stars are discussed separately in Sect. 4.2. If we disregard the three objects listed above, the correlation in Fig. 3 is again highly significant, with $\bar{R}=0.91$.

Since both the blue-peak velocity of the [N II] profile and the [O I] blue-edge velocity have been found to depend on the 


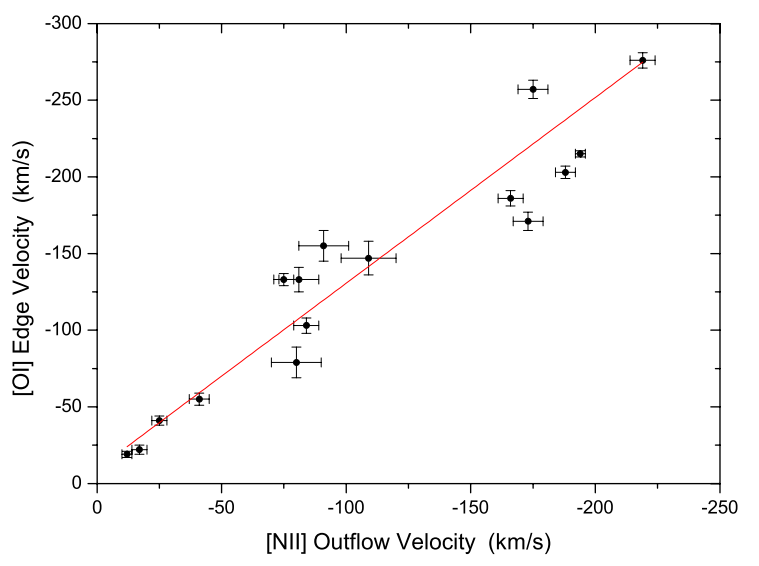

Fig. 4. Blue-edge velocities of the of the [O I] $630 \mathrm{~nm}$ lines as a function of the projected wind velocities indicated by the blue peak of the [N II] $658.3 \mathrm{~nm}$ line, and the corresponding linear regression line.

inclination, there has to be some mutual correlation between these two quantities. This is illustrated in Fig. 4. Since the [O I] edge measures the maximal outflow velocity, it is expected to be systematically higher than the [N II] peak velocity. This is confirmed by Fig. 4, where the [O I] edge velocities are on average $25 \%$ higher than the [N II] peak velocities. The individual points in Fig. 4 deviate from the mean relation on average by only about $20 \mathrm{~km} \mathrm{~s}^{-1}$. This value is not much more than the differences expected from the measuring errors alone $(\bar{R}=0.96$ for this correlation).

\subsection{Weak-wind stars}

As noted above and shown in Fig. 3, the three stars BP Tau, GM Aur, and TW Hya were found to have [O I] absolute edge velocities $\leq 31 \mathrm{~km} \mathrm{~s}^{-1}$ in spite of $\cos i$ values $>0.6$. In all three cases, the observed [O I] edge velocity differs from the mean relation by more than $20 \sigma$. All three stars have only very weak forbidden lines without detectable broad components. In contrast to the edge-on CTTSs with narrow [O I] lines, these three stars have no detectable jets (e.g., Azevedo et al. 2007). Moreover, except for the blueshifted absorption component of the He I $1083 \mathrm{~nm}$ line of TW Hya, wind absorption features of the permitted lines are very weak or absent in the spectra of these stars. GM Aur and TW Hya are relatively evolved pre-main-sequence stars with estimated evolutionary ages of, respectively, 7.4 Myr and $\approx 10 \mathrm{Myr}$ (Bertout et al. 2007; Webb et al. 1999). In the spectrum of GM Aur, the emission lines sometimes almost disappear. During such periods, GM Aur has the spectral properties of a weak-line TTS (Edwards et al. 2006). BP Tau has a lower age (3.2 Myr, according to Bertout et al. 2007). But, according to Dutrey et al. (2003), this star is "a transient object in the process of clearing its disk". Kwan et al. (2007) tentatively explained the absence of wind absorption features in the spectrum of BP Tau by an inclination effect. However, as shown later in this section, other stars with similar or higher inclinations do have wind absorption components, and an inclination effect cannot explain the weak and narrow forbidden lines of BP Tau.

In view of the low observed [O I] edge velocity and the weak wind absorption features BP Tau, GM Aur, and TW Hya, which in the following are denoted as "weak-wind stars", are indicated by open symbols in the following plots, and they are not included in graphs showing deprojected wind velocities.

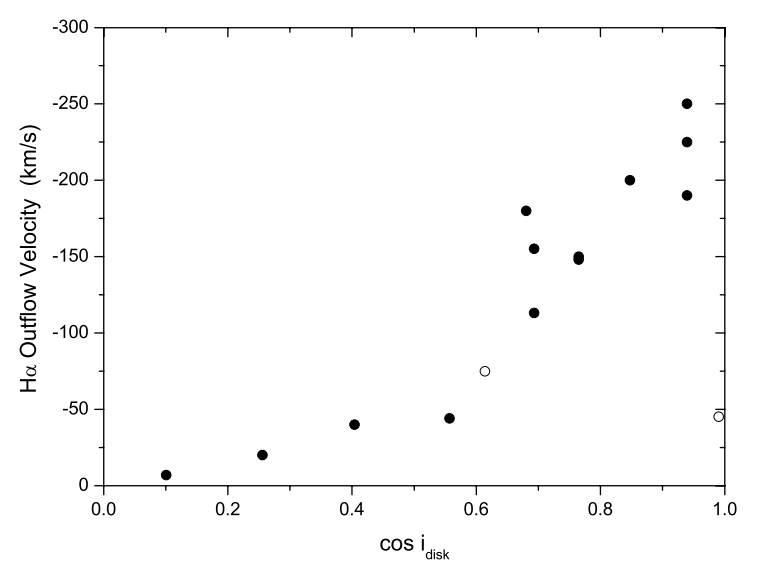

Fig. 5. Projected wind velocities inferred from the blueshifted absorption component of the $\mathrm{H} \alpha$ line as a function of the cosine of the disk inclination. Because of the time variability of the $\mathrm{H} \alpha$ profiles, errors cannot be reliably estimated for the values given for this feature. Therefore, error bars are omitted in this plot. The open symbol refers to GM Aur. Note the weak dependence on $\cos i$ of the outflow velocity for $\cos i<0.7$.

Some other stars in Table 1 may also belong to the weakwind category, although a confident assignment to this class is only possible for the three objects mentioned above, for which reliable inclination data are available. The most likely additional candidate for the weak-wind group in our sample is the CTTS GQ Lup, which is one of the best studied TTSs. Highresolution spectra of this object cover more than 35 years. All show a typical CTTS spectrum with significant mass accretion. However, the star again belongs to the more evolved TTSs (e.g., Donati et al. 2012; Johns-Krull et al. 2013). No reliable disk inclination has been reported for GQ Lup. But its rotation-based inclination $\left(27^{\circ}\right)$ is low and therefore probably not dramatically wrong (see Sect. 3). In spite of its likely low inclination, GQ Lup has a very low blueshift of its [O I] emission edge, and its [O I] $630 \mathrm{~nm}$ emission strength $(E W \approx 80 \mathrm{~m} \AA)$ is the lowest of all objects in Table 1.

\subsection{Wind absorption features of permitted emission lines}

Other spectral features usually ascribed to the winds of CTTSs are the blueshifted absorption components of strong permitted emission lines. Thus, their observed velocities are expected to be correlated with the inclination, too. As shown in Fig. 5, the central velocity of the blueshifted absorption component in the blue wings of the $\mathrm{H} \alpha$ lines does, as expected, increase with $\cos \left(i_{\text {disk }}\right)$. However, the relation observed in Fig. 5 clearly differs from the one found for the forbidden lines, as plotted in Figs. 2 and 3. While for the forbidden lines the apparent velocity increases more or less linearly with $\cos i$, in Fig. 5 the projected velocity remains low for approximately $\cos i<0.7$ (corresponding to $i>45^{\circ}$ ) and increases steeply for smaller inclinations. Since an absorption feature requires that the line-of-sight passes through the absorbing volume, as well as through the emission source, such behavior is to be expected for a wind that is restricted to a polar flow cone (see, e.g., Edwards et al. 2006). For a point-like continuum emission source, Fig. 5 can be explained by a polar flow cone with an opening angle of $45^{\circ}$. However, depending on the relative sizes and distance of the absorbing and continuum emitting volumes, Fig. 5 would also be consistent with a much smaller opening angle and an extended continuum emission region. 


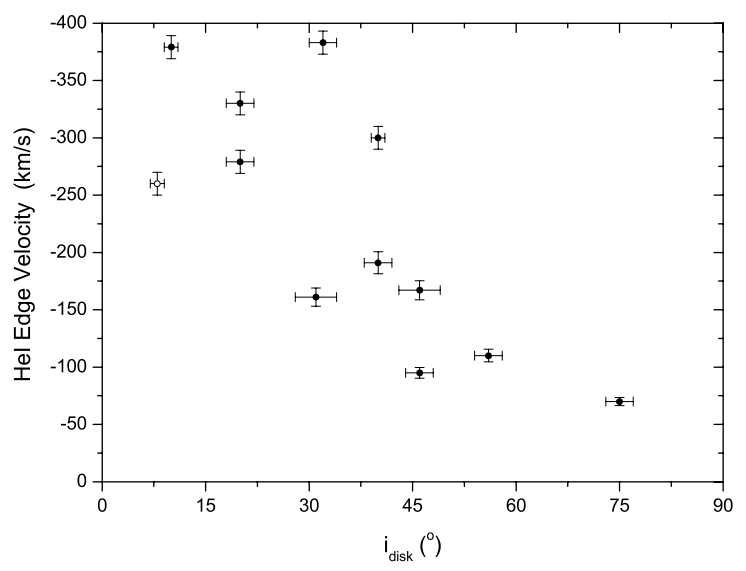

Fig. 6. Projected wind velocities inferred from the blue edge of the wind absorption component of the He I $1083 \mathrm{~nm}$ line as a function of the disk inclination. The open symbol refers to TW Hya.

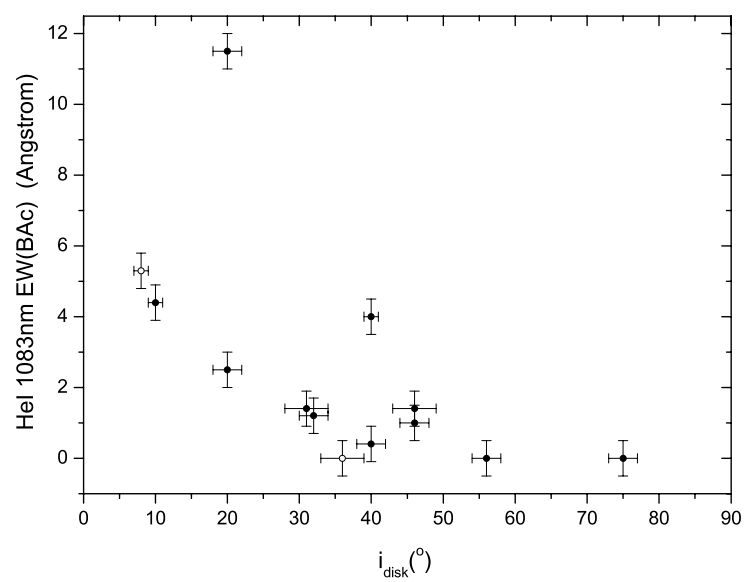

Fig. 7. Equivalent width below the continuum of the blueshifted absorption component of the $\mathrm{He} \mathrm{I} 1083 \mathrm{~nm}$ line profile as a function of the disk inclination. The two open symbols refer to BP Tau and TW Hya.

Among the permitted lines in TTS spectra, the He I $1038 \mathrm{~nm}$ line, which has a metastable lower level, is particularly well suited for studying the kinematics of absorbing gas flows. Therefore, we compare in Fig. 6 the apparent wind velocities inferred from this line with the observed disk inclinations. Since the absorption by this line usually covers a broad velocity range, and since we are mainly interested in the terminal velocity of the wind, we use the blue-edge velocity of the absorption component for this line. To have a consistent and reproducible measure, we plot the velocity where the blue wing of the wind absorption reaches $25 \%$ of the central depth in the profiles published by Edwards et al. (2006). The sample for which we have data on this spectral feature, as well as on disk inclinations, only contains one object with $\cos i<0.5$. Therefore, we use the disk inclination angle in Fig. 6, rather than its cosine, as the abscissa. The figure again demonstrates that there is a correlation. The data points are consistent with a steep increase in the apparent wind velocity for approximately $i<45^{\circ}$ (as observed for the $\mathrm{H} \alpha$ wind absorption), but the lack of more data at high inclinations does not allow us to prove this behavior independently in the case of the He I wind absorption alone. Interestingly, the He I edge velocity of the weak-wind star TW Hya $\left(-260 \mathrm{~km} \mathrm{~s}^{-1}\right.$, $i_{\text {disk }}=8^{\circ}$ ) also fits the trend shown in the figure. (For the other weak-wind objects, the corresponding spectral feature is very weak or absent.)

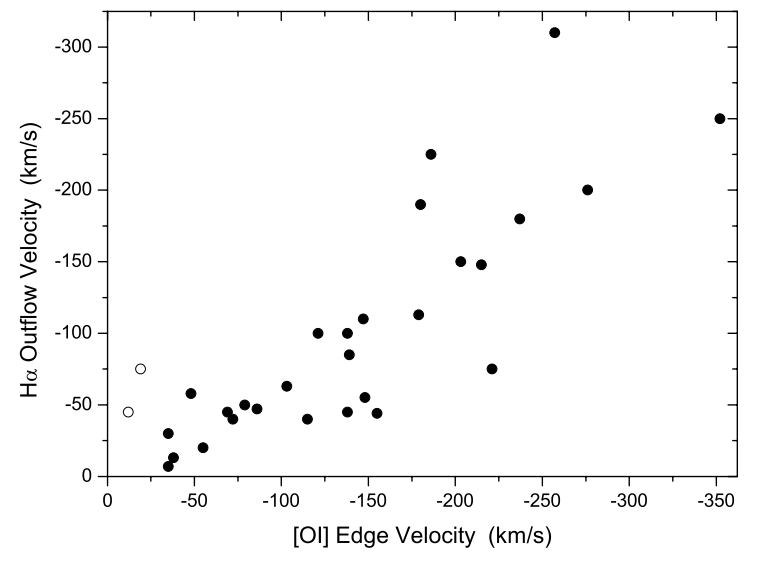

Fig. 8. Projected wind velocities inferred from the blueshifted absorption component of the $\mathrm{H} \alpha$ line as a function of the projected wind velocity indicated by the blue edge of the [O I] $630 \mathrm{~nm}$ line. The open symbols refer to GM Aur and TW Hya.

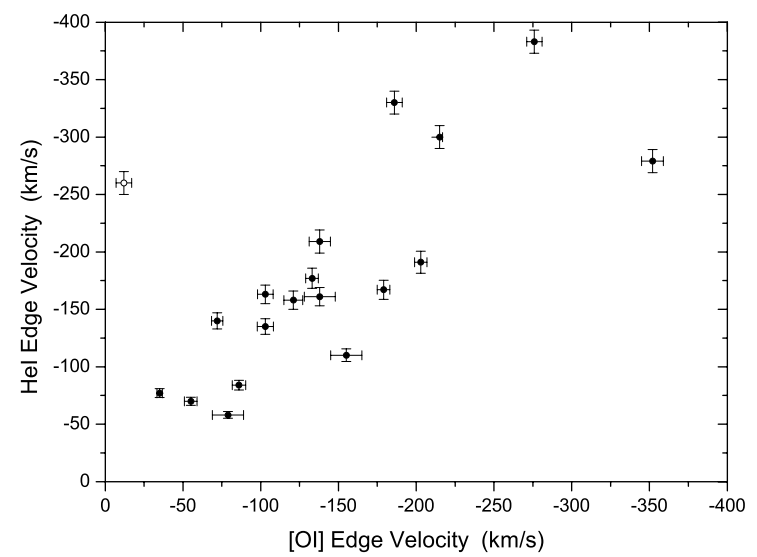

Fig. 9. Projected wind velocities inferred from the blue edge of the wind absorption component of the He I $1083 \mathrm{~nm}$ line as a function of the projected wind velocity indicated by the blue edge of the [O I] $630 \mathrm{~nm}$ line. The open symbol refers to TW Hya.

A related correlation with the disk inclination is shown in Fig. 7, where we plot the equivalent width below the continuum of the blueshifted absorption component of the He I $1083 \mathrm{~nm}$ line. The corresponding data are again taken from Edwards et al. (2006). The uncertainty of the EW values has been estimated from the scatter of those cases where observations for more than one epoch have been reported. The absence of a significant absorption for inclinations $>45^{\circ}$ again supports the assumption of a cone-like outflow geometry with an opening angle $\leq 45^{\circ}$. The data point of the weak-wind star TW Hya $(E W=5.3 \AA$, $i_{\text {disk }}=8^{\circ}$ ) again fits the trend indicated in Fig. 7 .

Since the velocities of the wind absorption features and those of the forbidden lines were both found to be correlated with the inclination, we also expect correlations between the forbiddenline velocities and the wind absorption. This is confirmed in Figs. 8 and 9, where we plot our measures for the wind velocities of the permitted lines as a function of the [O I] outflow velocities. Interestingly, for the He I absorption edge, we obtain on average fairly similar projected outflow velocities to those for the $[\mathrm{O} \mathrm{I}]$ emission line edge, although these features can hardly be produced in the same volumes. The central velocities of the $\mathrm{H} \alpha$ absorption features have on average only about two thirds of the [O I] edge velocities. Although at least part of the correlations indicated in Figs. 8 and 9 certainly result from 


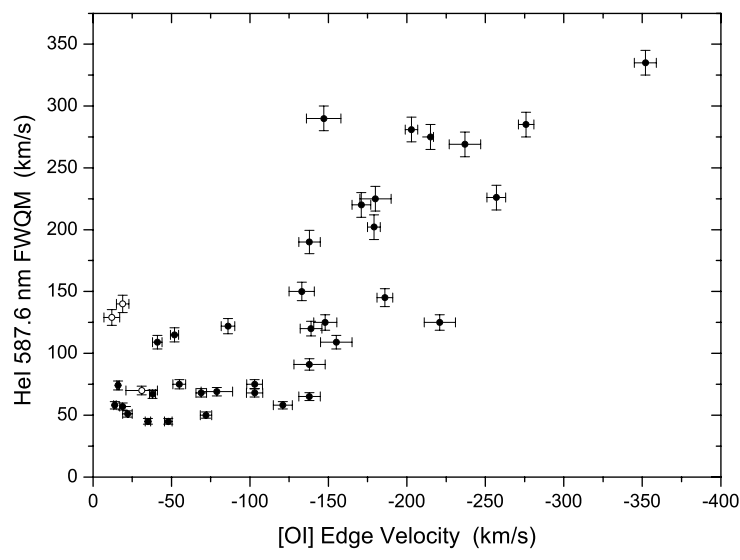

Fig. 10. Full width at one quarter of the maximum of the He I $587.6 \mathrm{~nm}$ emission line as a function of the projected wind velocity indicated by the blue edge of the [O I] $630 \mathrm{~nm}$ line. The three weak-wind objects are again indicated by open symbols.

an inclination effect, a contribution from a correlated intrinsic wind velocity cannot be excluded. But in both cases it is clear from the figures that the winds seen in both the forbidden lines and the permitted lines are physically related, in spite of the very different conditions for forming these different spectral features.

\subsection{The He I $587.6 \mathrm{~nm}$ line width}

Another permitted line that has been discussed extensively in the context of the polar winds of CTTSs is the He I $587.6 \mathrm{~nm}$ emission feature (see, e.g., Beristain et al. 2001; Kwan \& Fischer 2011). Like the forbidden lines, this He I line often consists of both a broad and a narrow component. The broad components of the He I $587.6 \mathrm{~nm}$ line have widths that are comparable to those of the broad components of the forbidden lines. However, the profiles of the broad components of the helium lines are quite different from those of the broad components of the forbidden lines. While the forbidden lines often have a steep blue wing and no red wing, the broad components of the helium lines are in most cases essentially symmetric with a Gaussian-like profile. The narrow components of the helium lines are also symmetric and slightly broader than the corresponding forbidden line features. Also in contrast to the corresponding forbidden-line broad components, the broad helium-line components are only modestly blueshifted relative to the systemic velocity by about $75 \mathrm{~km} \mathrm{~s}^{-1}$ on average.

The broad helium emission component is often assumed to form in the T Tauri winds (e.g., Kwan \& Fisher 2011). Therefore, we compared the observed widths of the He I $587.6 \mathrm{~nm}$ line with the disk inclinations. As a measure of the width, we used the full width of the line at a quarter of the maximal intensity (FWQM) of the total line. For the 15 objects where disk inclination data and the He I $587.6 \mathrm{~nm}$ line widths are listed in Table 1, we find a decrease in the line width with increasing inclination, which is to be expected when the width is related to a polar wind. However, the scatter around the mean relation is too large to regard this result as conclusive.

On the other hand, a more convincing correlation is found when the FWQM of the total He I emission is compared with the [O I] edge velocity (Fig. 10). Although the scatter of the individual data points is large, the relatively high number of data points makes the correlation significant. Since (as shown above) the $[\mathrm{O}$ I] velocity is well correlated with the inclination, Fig. 10 may provide indirect evidence for a relation between the maximal velocity observed in the He I $587.6 \mathrm{~nm}$ line $^{2}$ and the inclination, which supports a wind origin of at least part of this line emission. But the sample for which these informations are available is too limited to derive firm results. No significant correlations between the inclination and the FWHM of the broad component of the He I line (again listed in Beristain et al. 2001) and no correlation between the inclination and the intensity ratio between broad and narrow He I line components (BC/NC) could be detected, in contrast to the result for the forbidden lines. However, we suspect that such correlations do exist and could be established with larger samples.

A closer look at the individual lines reveals a qualitatively different dependence on inclination for the forbidden-line and helium-line profiles. For the forbidden lines, the broad component simply becomes narrower with increasing inclination and finally merges into the narrow component. In the case of the helium lines, broad components are found at all inclinations, although their widths and strengths decrease with increasing inclination. A good example for the different behaviors of the forbidden lines and the helium lines is the spectrum of the edgeon object HH30* $\left(i=82^{\circ}\right)$, which shows a weak broad component of the He I $587.6 \mathrm{~nm}$ line $\left(F W H M \approx 140 \mathrm{~km} \mathrm{~s}^{-1}\right)$, while a broad component of the [O I] $630 \mathrm{~nm}$ line is not detectable (Appenzeller et al. 2005). Similar behavior is seen in the spectrum of AA Tau $\left(i=75^{\circ}\right)$.

\section{Negative results}

In addition to those discussed in Sect. 4, we searched for correlations between the disk inclinations and various other spectral properties, such as the strength and width of selected emission lines or line components, the veiling, and the presence and strength of redward-displaced (accretion) absorption features. Apart from some quantities that are related to the line profile data discussed in Sect. 4, no other significant correlations were detected. In contrast to a tentative result noted earlier (Appenzeller et al. 2005) and expectations from the theory (Kurosawa et al. 2006), no significant correlation could be found between the $\mathrm{H} \alpha$ equivalent width and the inclination in our larger sample with more reliable inclination angles. If such a relation exists, it is obliterated by the very large scatter in the equivalent widths of the individual stars and by the variability of this line. Larger samples will probably be needed to detect a corresponding correlation by statistical means.

A search for a correlation between the H $\alpha$ FWHM line width and the inclination also ended inconclusively. Although the line widths were found to be on average lower for disk inclinations $>50^{\circ}$, the scatter of the individual values is very large, and the result is far from being statistically significant.

We did not detect any correlation between the disk inclination and the presence or strength of the redshifted ("YY Orionis") absorption components of the He I $1083 \mathrm{~nm}$ line profiles observed by Edwards et al. (2006) and Fisher et al. (2008). Redshifted absorption components were found at all disk inclinations between $8^{\circ}$ and $75^{\circ}$, and no dependence of their strength on the inclination is evident from the available data. The lack of such a correlation may be explained by the dependence of the accretion flows on the geometry of the magnetic fields, which in many cases is known to be tilted relative to the rotation

2 Or some other measure of the broad-component width of the He I $587.6 \mathrm{~nm}$ line, such as the parameter $V_{\text {bwing }}$ listed by Beristain et al. (2001). 


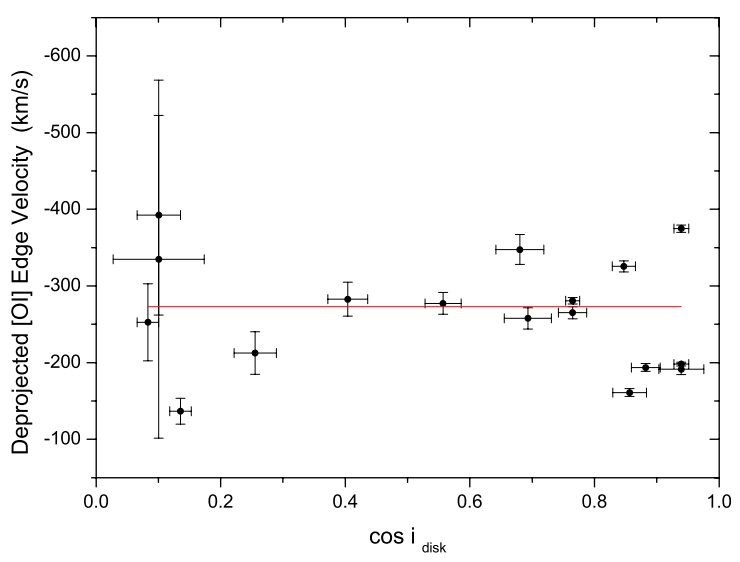

Fig. 11. Deprojected outflow velocities, as derived from the blue edge of the $[\mathrm{O} \mathrm{I}]$ emission line, as a function of the cosine of the disk inclination.

axis (e.g., Bouvier et al. 2007; O'Sullivan et al. 2005). Moreover, the redshifted absorption components are known to show strong time variations, which may mask any correlation with $i$.

\section{Deprojected wind velocities}

Assuming that the polar outflows from the CTTSs are normal to the disk planes, one can derive the true, deprojected flow velocities by dividing the observed velocities by the cosine of the corresponding disk inclination, as listed in Table 1. We carried out these calculation for the [O I], [N II], $\mathrm{H} \alpha$, and $\mathrm{He}$ I velocities where disk inclination data are available in Table 1. Figure 11 gives the resulting deprojected [O I] edge velocities as a function of $\cos i$. For the reasons outlined in Sect. 4, the three weakwind TTSs have been omitted in this figure. The large error bars near the left margin of the figure result from the $(\cos i)^{-1}$ magnification of all errors for high inclinations. As shown by Fig. 11, the deprojected velocities scatter around a horizontal line. No systematic dependence of the deprojected velocities on the inclination is detected, which appears to confirm the correctness of the deprojection procedure. An error-weighted least square solution results for the complete sample of Fig. 11 in a deprojected flow velocity of $273 \pm 14 \mathrm{~km} \mathrm{~s}^{-1}$. A simple average of all deprojected velocities of Fig. 11 gives a flow velocity of $262 \pm 18 \mathrm{~km} \mathrm{~s}^{-1}$. An average of the (possibly more reliable) data for the 13 objects with $i<80^{\circ}(\cos i>0.17)$ gives $258 \pm 18 \mathrm{~km} \mathrm{~s}^{-1}$. The median deprojected velocity of both samples is $258 \mathrm{~km} \mathrm{~s}^{-1}$. All deprojected velocities range between $137 \mathrm{~km} \mathrm{~s}^{-1}<v_{\text {depr }}<392 \mathrm{~km} \mathrm{~s}^{-1}$. If we disregard the less certain values for $i>80$ degrees, the range narrows to the interval $161 \mathrm{~km} \mathrm{~s}^{-1}$ to $374 \mathrm{~km} \mathrm{~s}^{-1}$. Neglecting the results for HV Tau C and KH15D, which have errors of the deprojected velocities exceeding $100 \mathrm{~km} \mathrm{~s}^{-1}$, we find that $80 \%$ of all flow velocities are in the range between $190 \mathrm{~km} \mathrm{~s}^{-1}$ and $350 \mathrm{~km} \mathrm{~s}^{-1}$.

Deprojected velocities of the [N II] blue emission peaks are plotted in Fig. 12. Because these values correspond to to a mean flow velocity (while the [O I] data correspond approximately to the maximal flow velocity), the [N II] velocities are on average lower by about $20 \%$. For the [N II] line, the error-weighted mean and the simple average for all objects is $196 \pm 16 \mathrm{~km} \mathrm{~s}^{-1}$ in both cases. If only the objects with $i<80$ degrees are averaged, we get $205 \pm 14 \mathrm{~km} \mathrm{~s}^{-1}$. The median value is $195.5 \mathrm{~km} \mathrm{~s}^{-1}$.

In Fig. 13 we give deprojected velocities of the blue absorption component of the $\mathrm{H} \alpha$ lines. As discussed in Sect. 4, the velocities of this spectral feature behave differently for inclinations below and above about $45^{\circ}$. This bimodality is confirmed

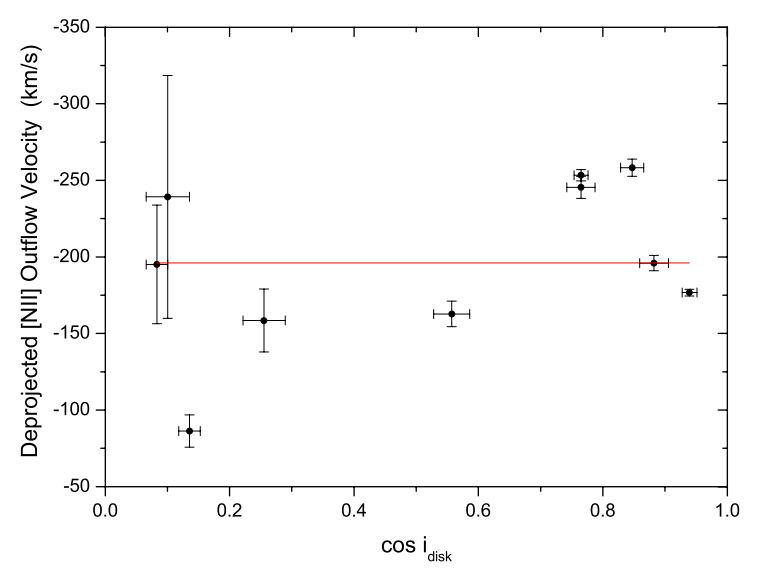

Fig. 12. Deprojected outflow velocities, as derived from the blue peak of the $[\mathrm{N} \mathrm{II}]$ emission lines, as a function of the cosine of the disk inclination.

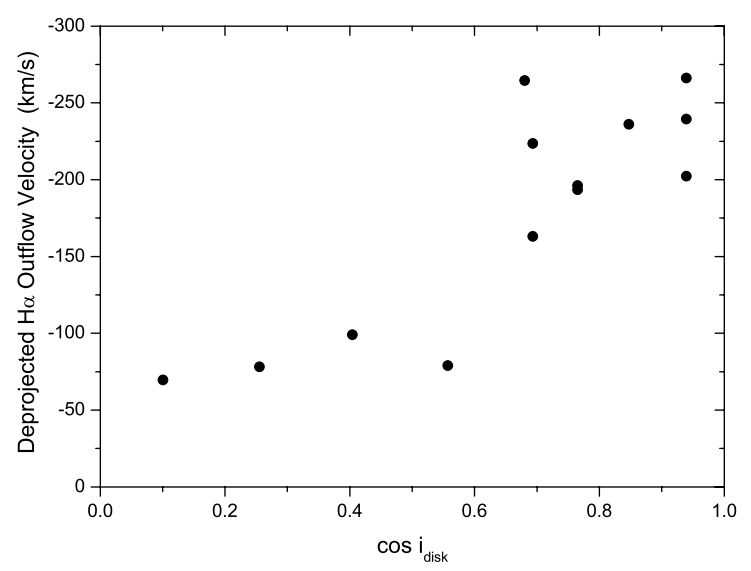

Fig. 13. Deprojected outflow velocities indicated by the blueshifted absorption components of the $\mathrm{H} \alpha$ line profiles as a function of the cosine of the disk inclination.

by Fig. 13, which indicates a low deprojected velocity for high inclinations ( $\cos i<0.68)$ and high velocities for smaller inclinations. The pattern is again consistent with the assumption of a fast polar wind with a cone-like geometry and an opening angle $\leq 45^{\circ}$. The mean flow velocity derived from the $\mathrm{H} \alpha$ blue absorption components with $\cos i_{\text {disk }}>0.68$ is $224 \pm 11 \mathrm{~km} \mathrm{~s}^{-1}$, which is slightly higher than the average [N II] (peak) flow velocity, but lower than the mean deprojected [O I] flow velocity.

Deprojected flow velocities were also calculated for the blueedge velocities of the He I $1083 \mathrm{~nm}$ absorption components. The scatter of the resulting data with $\cos i>0.68$ is larger than in the case of the [O I] emission line edges, but the mean value $292 \pm 33 \mathrm{~km} \mathrm{~s}^{-1}$ is (within the error limits) compatible with the [O I] result.

\section{Correlations with other stellar parameters}

The scatter of the deprojected flow velocities of the forbidden lines is clearly greater than can be explained by the observational errors alone. Thus, assuming that the disk inclination errors cited in the literature are correct, we have to conclude that significant, real differences exist between the outflow velocities of the individual objects. To clarify the origin of these differences, we searched for correlations between the individual deprojected velocities and other stellar properties. In detail, we checked for possible correlations between these velocities and 


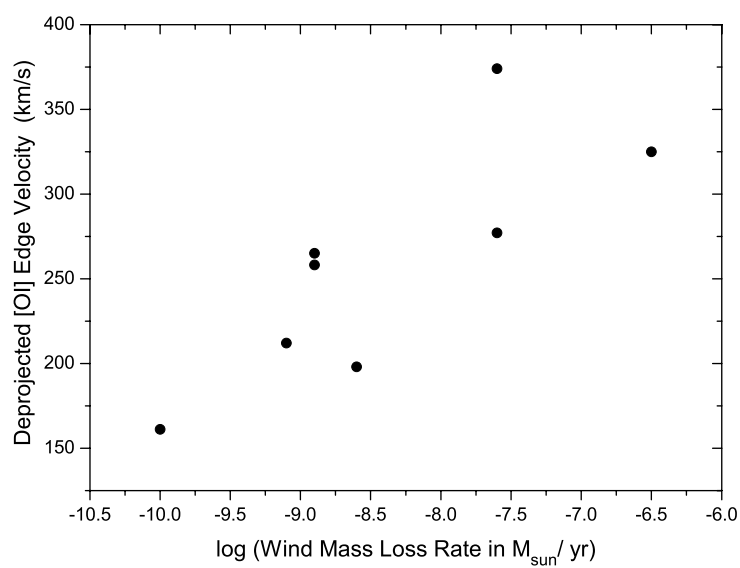

Fig. 14. Deprojected wind flow velocities inferred from the blue edge of the [OI] $630 \mathrm{~nm}$ lines as a function of the logarithm of the wind mass loss rate.

the objects' escape velocities, photospheric radii, rotation periods, magnetic field data, spectral veiling, mass accretion rates, wind mass loss rates, and various line profile properties. No convincing correlations could be found. A trend, shown in Fig. 14, was found for the relation between the deprojected [O I] velocity and the wind mass loss rate as listed by Hartigan et al. (1995). The flow velocity appears to increase systematically from about $200 \mathrm{~km} \mathrm{~s}^{-1}$ at $\dot{M}=-10^{-10} M_{\odot} / \mathrm{yr}$ to $\approx 400 \mathrm{~km} \mathrm{~s}^{-1}$ at about $\dot{M}=-3 \times 10^{-7} M_{\odot} /$ yr. However, this result is only based on eight data points. Perhaps more remarkable may be that the velocity change is only a factor two, while the mass loss rate changes by about $3 \times 10^{3}$.

That no other correlations of the deprojected velocity with stellar parameters could be detected does not rule out that such correlations exist. In most cases the detection of significant correlations was precluded by the smallness of our samples and/or by too large errors of the input data. Additional data will be needed to find correlations with other stellar parameters and to clarify the origin of the differences in outflow velocities.

\section{Conclusions}

The results described in the previous sections show that various properties of the spectra of CTTSs are correlated with the inclinations of their associated circumstellar disks. No such correlations could be detected for inclinations derived from the rotational data of these stars, and the correlation between the rotation-based inclinations and the disk inclinations is rather poor. This seems to indicate that only the disk inclinations provide a good measure of the true orientations of the TTS systems, while the values derived from stellar rotation data are not yet accurate enough for such studies.

The most conspicuous correlations with the disk inclination are observed for the profiles of the forbidden emission lines and for the velocities of the wind absorption features of some permitted lines. The data support the assumption of cone-like fast polar winds with opening angles $\leq 45^{\circ}$. A correlation is also indicated between the disk inclination and the width of the He I $587.6 \mathrm{~nm}$ emission line. This supports earlier suggestions that this line is at least in part related to the T Tauri winds.

Using the disk inclination values, true (deprojected) wind flow velocities were calculated. From the blue edge of the [O I] emission, we find mean flow velocities of about $260 \mathrm{~km} \mathrm{~s}^{-1}$, with the well determined individual values ranging between
$161 \mathrm{~km} \mathrm{~s}^{-1}$ and $374 \mathrm{~km} \mathrm{~s}^{-1}$. The blue edges of the wind absorption features of the He I $1083 \mathrm{~nm}$ line give similar mean values, although the scatter of the individual velocities is greater. The deprojected central velocities of the $\mathrm{H} \alpha$ wind absorption components have velocities that are similar to or slightly larger than those derived from the blueshifted emission peaks of the [N II] lines. The derived deprojected flow velocities differ among the observed objects. Correlations of these differences with other stellar parameters could not be reliably established with the samples that are presently available. Larger samples, which will probably become available with future ALMA observations of TTS disks, may provide answers to this question.

Acknowledgements. We would like to thank the referee, Dr. Suzan Edwards, for valuable comments and helpful suggestions.

\section{References}

Akeson, R. L., Millan-Gabet, R., Ciardi, D. R., et al. 2011, ApJ, 728, 96 Alencar, S. H. P., \& Basri, G. 2000, AJ, 119, 1881

Alencar, S. H. P., \& Batalha, C. 2002, ApJ, 571, 378

Alencar, S. H. P., Johns-Krull, C. M., \& Basri, G. 2001, AJ, 122, 3335

Andrews, S. M., \& Williams, J. P. 2007, ApJ, 659, 705

Appenzeller, I., \& Wagner, S. 1989, A\&A, 225, 432

Appenzeller, I., Jankovics, I., \& Jetter, R. 1986, A\&AS, 64, 65

Appenzeller, I., Bertout, C., \& Stahl, O. 2005, A\&A, 434, 1005

Azevedo, R., Folha, D. F. M., Gameiro, J. F., et al. 2007, ApJ, 670, 1234

Beristain, G., Edwards, S., \& Kwan, J. 2001, ApJ, 551, 1037

Bertout, C., Basri, G., \& Bouvier, J. 1988, ApJ, 330, 350

Bertout, C., Siess, L., \& Cabrit, S. 2007, A\&A, 473, L21

Boesgaard, A. M. 1984, AJ, 89, 1635

Böhm, K.-H., \& Solf, J. 1994, ApJ, 430, 277

Bouvier, J., Covino, E., Kovo, O., et al. 1995, A\&A, 299, 89

Bouvier, J., Alencar, S. H. P., Boutelier, T., et al. 2007, A\&A, 463, 1017

Broeg, C., Schmidt, T. O. B., Guenther, E., et al. 2007, A\&A, 468, 1039

Camenzind, M. 1990, Rev. Mod. Astron., 3, 234

Cox, A. W., Grady, C. A., Hammel, H. B., et al. 2013, ApJ, 762, 40

Curiel, S., Raga, A., Raymond, J., et al. 1997, AJ, 114, 2736

Donati, J.-F., Gregory, S. G., Alencar, S. H. P., et al. 2012, MNRAS, 425, 2948

Dutrey, A., Guilloteau, S., \& Simon, M. 2003, A\&A, 402, 1003

Edwards, S., Hartigan, P., Ghandour, L., \& Andrulis, C. 1994, AJ, 108, 1056

Edwards, S., Fischer, W., Hillenbrand, L., \& Kwan, J. 2006, ApJ, 646, 319

Fischer, W., Kwan, J., Edwards, S., \& Hillenbrand, L. 2008, ApJ, 687, 1117

Guilloteau, S., Dutrey, A., Piétu, V., \& Boehler, Y. 2011, A\&A, 529, A105

Hamann, F. 1994, ApJS, 93, 485

Hamann, F., \& Persson, S. E. 1992, ApJS, 82, 247

Hamilton, C. M., Herbst, W., Mundt, R., et al. 2003, ApJ, 591, L45

Hartigan, P., Edwards, S., \& Ghandour, L. 1995, ApJ, 452, 736

Hartmann, L., Hewett, R., \& Calvet, N. 1994, ApJ, 426, 669

Herczeg, G. J., Najita, J. R., Hillenbrand, L. A., \& Pascucci, I. 2007, ApJ, 670, 509

Hirth, G. H., Mundt, R., \& Solf, J. 1997, A\&AS, 126, 437

Isella, A., Carpenter, J. M., \& Sargent, A. I. 2010, ApJ, 714, 1746

Johns-Krull, C. M., \& Gafford, A. D. 2002, ApJ, 573, 685

Johns-Krull, C. M., Chen, W., Valenti, J. A., et al. 2013, ApJ, 765, 11

Krautter, J., Appenzeller, I., \& Jankovics, I. 1990, A\&A, 236, 416

Kurosawa, R., Harries, T. J., \& Symington, N. H. 2006, MNRAS, 370, 580

Kurosawa, R., Romanova, M. M., \& Harries, T. J. 2011, MNRAS, 416, 2623

Kwan, J., \& Fischer, W. 2011, MNRAS, 411, 2383

Kwan, J., Edwards, S., \& Fischer, W. 2007, ApJ, 657, 897

Kwon, W., Looney, L. W., \& Mundy, L. G. 2011, ApJ, 741, 3

Muzerolle, J., Calvet, N., \& Hartmann, L. 2001, ApJ, 550, 944

O'Sullivan, M., Truss, M., Walker, Ch., et al. 2005, MNRAS, 358, 632

Ratzka, T., Chen, W., Valenti, J. A., et al. 2009, A\&A, 502, 623

Roccatagliata, V., Ratzka, T., Henning, T., et al. 2011, A\&A, 534, A33

Schegerer, A. A., Wolf, S., \& Hummel, C. A. 2009, A\&A, 502, 367

Shevchenko, V. S., Grankin, K. N., Mel'Nikov, S. Yu., et al. 1998, Astr. Lett., 24,528

Stapelfeldt, K. R., Krist, J. E., Menard, F., et al. 1998, ApJ, 502, L65

Stapelfeldt, K. R., Ménard, F., Watson, A. M., et al. 2003, ApJ, 589, 410

Stempels, H. C., \& Piskunov, N. 2002, A\&A, 391, 595

Tanii, R., Itoh, Y., Kudo, T., et al. 2012, PASJ, 64, 124

Weaver, W. B. 1987, ApJ, 319, L89

Webb, R. A., Zuckerman, B., Platais, I., et al. 1999, ApJ, 512, L63

Yorke, H. W., Bodenheimer, B., \& Laughlin, G. 1993, ApJ, 411, 274 